\title{
IVIA - Ibero-American VLBI Initiative -Progress on the Brazilian side
}

\author{
JACQUES R.D. LEPINE, JEAN PIERRE RAULIN, TANIA DOMINICI, CARLOS \\ GUILLERMO G. DE CASTRO, FERNANDO VIRGILIO ROIG, ANDRÉ WIERMANN, \\ MARCELO BORGES FERNANDES, YASUCHI HADANO, FATIMA SALETE CORRERA, \\ WESLEY BECCARO, MARCOS AURELIO LUQUEZE, ANTONIO SANDRO VERRI, \\ DANILO CESAR ZANELLA, KARIN MENÉNDEZ-DELMESTRE, THIAGO SIGNORINI \\ GONÇALVES, ULISSES BARRES DE ALMEIDA \& MILITÃO VIEIRA FIGUEREDO
}

\begin{abstract}
IVIA is a joint initiative of at least 8 Latin-American countries plus Portugal and Spain to make good use of large telecommunications antennas that are out of service in these countries, because most international communications are now using submarine cables. The first step will be to refurbish the antennas and then to start doing single dish observations of radiosources. In a second step the antennas will be equipped with VLBI (Very Long Base Interferometry) equipment, to establish a VLBI network. This project will be able to effectively promote scientific integration in Ibero-America. The work is starting in several countries; here we present several scientific cases for the use of the antennas, and we report on the first Brasilian activities.
\end{abstract}

Key words: Ibero-American Initiative, IVIA, Telecommunication Antennas, Radio Astronomy, VLBI.

\section{INTRODUCTION}

There is a number of large telecommunication antennas in Latin America, plus Portugal and Spain (Ibero-America for short), with diameters up to $32 \mathrm{~m}$, that are now out of use, since the communications have been transferred to much more performant submarine optical fiber cables. In Brazil there are 3 antennas of this type; one of them, the Tanguá antenna, is shown in Figure 1. During a Regional meeting on VLBI (Very Long Base Interferometry) held in Mexico in February 2019, the IVIA (Initiative for VLBI in Ibero-America) was founded, with the plan to make use of these antennas in the frequency range 4 to $8 \mathrm{GHz}$, and to establish a regional VLBI network.

The foundation document of the collaboration was signed by representatives of 10 countries (Argentina, Brazil, Colombia, Costa Rica, Equador, Mexico, Peru, Portugal, Spain, Uruguay) and the representative of JIVE (Joint Institute for Very Long Baseline Interferometry European Research Infrastructure Consortium). Paraguay has recently joined the effort. The list of presently involved institutions and representative researchers is given in Appendix A. An attractive aspect of the IVIA project is that these countries, which have so many cultural similarities and ties, but have few joint scientific 


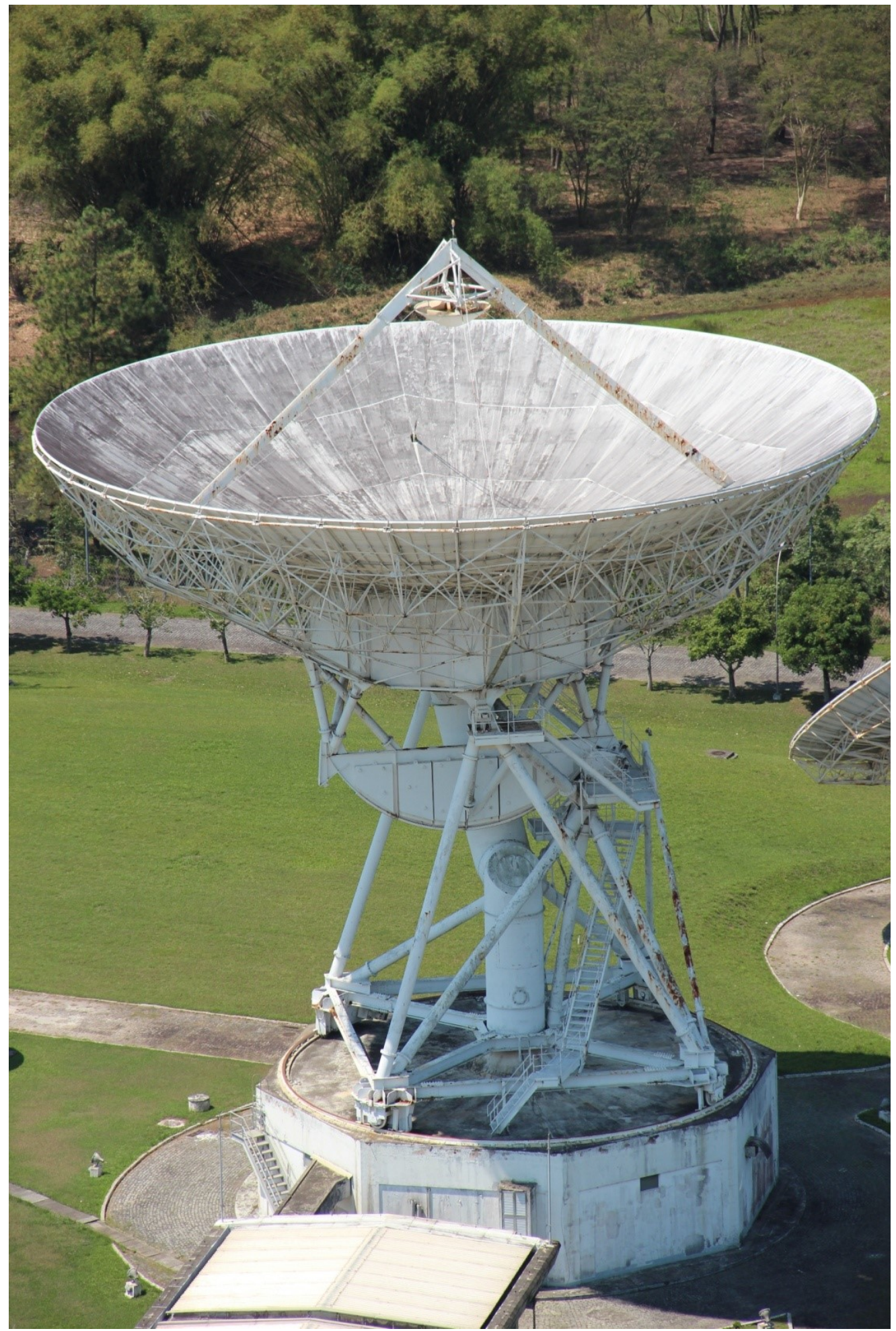

Figure 1. The Tanguá $32 \mathrm{~m}$ antenna (Rio de Janeiro State), planned to be part of IVIA. 
programs going on, have identified a motivating scientific objective to work together. Besides science, a number of other goals like outreach, attracting students to science, promotion of mobility of researchers and students, and others, have been discussed in the IVIA meeting. In the present work, we will to concentrate on two aspects; 1) the scientific and technological results that can be expected from IVIA, and 2) the technical side: what are the difficulties to be faced, including a report on a technical visit that has been made to the Tanguá antenna in Rio de Janeiro State, presented in Appendix B.

We must add, for start, that a similar effort in using telecommunication antennas for radio astronomy is going on in the African continent. They have some 20 antennas spread in many countries (Gaylard et al. 2011). In Nigeria, there are 3 antennas! Two of the African antennas are in more advanced state of progress than the South American ones. One is the South African $26 \mathrm{~m}$ dish in Hartebeesthoek, near Johanesburg, from where all the VLBI efforts started. The other one is the $32 \mathrm{~m}$ antena of Ghana which has been refurbished and has already participated in VLBI experiments ${ }^{1}$. Figure 2 indicates the positions of main antenas in African and American continents, and those belonging to Spain and Portugal. It can be seen that collaboration with African countries will be important in future to get a better coverage in the East-West direction.

\section{THE SCIENTIFIC CASE FOR IVIA}

In each participant country, the activities will be in two steps: 1) To transform the available antenna in a single dish working system, reaching an agreement with the company that owns the antenna, and refurbishing the antenna which has been out of use for many years.
Then, to install a 4 to $8 \mathrm{GHz}$ receiver with Total Power detection and a spectrometer, and use the antenna for astrophysical observations. 2) The second step is to acquire the equipment for VLBI observations: an atomic clock, a proper IF processor and a data storage system.

The scientific cases for the two phases are distinct. The single dish phase will probably occupy the first 2 years, but will have a continuation even after the VLBI equipment is available, since the VLBI activities will use only part of the antena time.

\section{Radio recombination lines}

There are types of observations which have been made with large antennas already for a few decades, but are still of great utility. The radio recombination lines observed in ionized gas (HII regions) are an example. These lines appear after the recombination of ions and electrons to form atoms. In the Bohr atom model the excited levels of $\mathrm{H}$ atom are labeled by their principal quantum number $n$. In a recombination process, an electron is captured at a high " $n$ " level and then cascades down to lower $n$, emitting line radiation at each step of the ladder. In the range of frequency from 4 to $8 \mathrm{GHz}$, all the lines between $117 \alpha(4.05 \mathrm{GHz})$ to $93 \alpha(8.04 \mathrm{GHz})$ are available. Recombination lines of Helium and of Carbon are situated close to the $\mathrm{H}$ lines. The recombination lines allow to determine physical parameters like temperature, density, velocity, $\mathrm{He} / \mathrm{H}$ and $\mathrm{C} / \mathrm{H}$ density ratios.

\section{Methanol masers}

We will focus now on a more recent lines of research. Observations of methanol masers at $6.668 \mathrm{GHz}$ are of particular interest for single dish observations, since these masers display intriguing periodic variability that is not

\footnotetext{
${ }^{1}$ https://www.nature.com/news/ghana-telescope-heralds-first-pan-african-array-1.21958.
} 


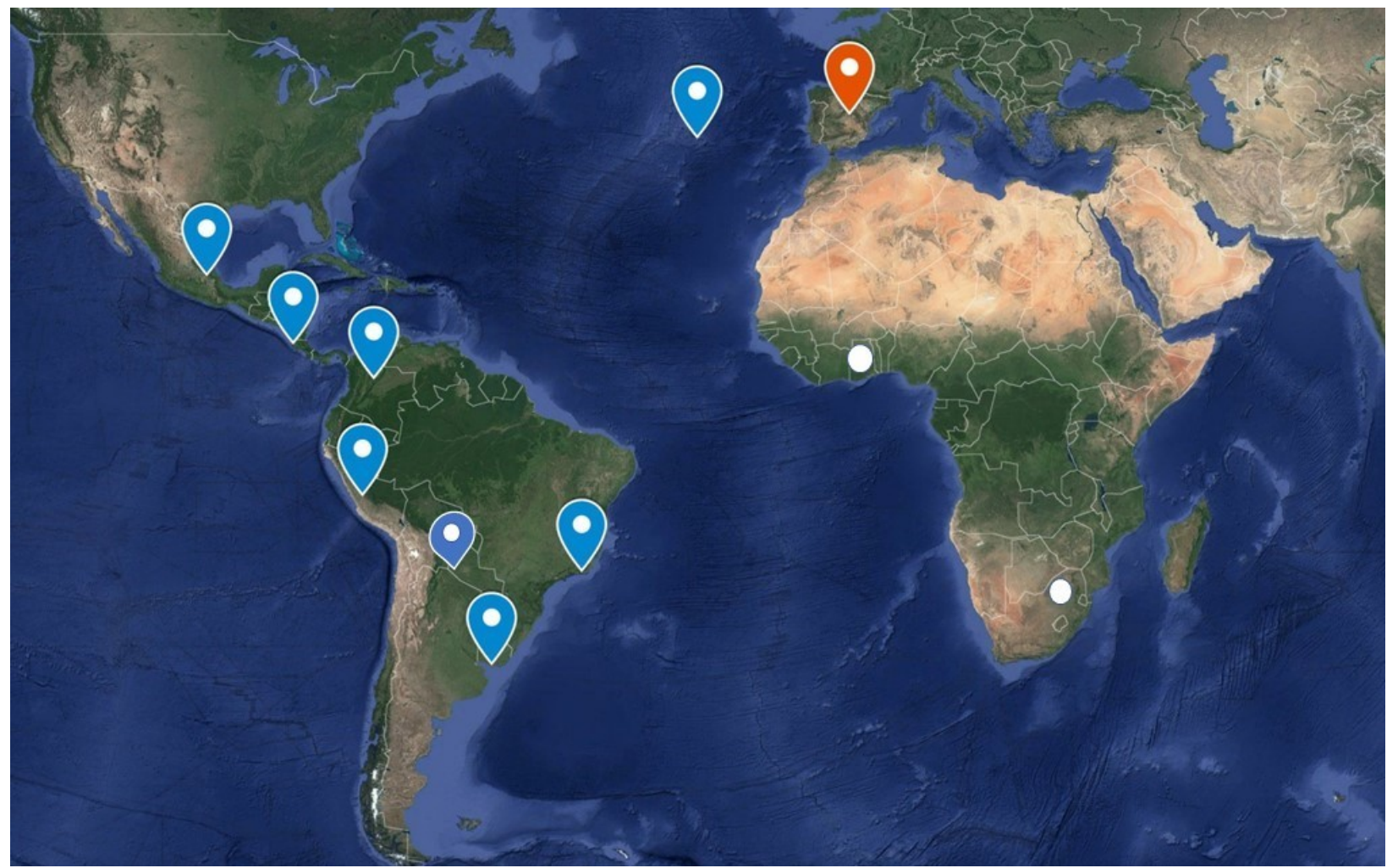

Figure 2. The position of the main antennas participating in IVIA. In South America, the antena of Paraguay has been added to the original map of IVIA. In Brazil, only the Tanguá antenna is shown, although another antena at Morungaba, São Paulo State, might participate in future. The antena in the ocean is the one of Azores, belonging to Portugal. The Yebes Antenna in Spain is shown in red; it is currently participating in VLBI observations with the European network. Two antennas are shown in Africa, in Ghana (Kuntunse Satellite Earth Station) and South Africa (Hartebeesthoek, near Johanesburg).

yet understood. We reproduce in Figure 2 the time variation of 2 of these sources, taken from Olech et al. (2019). The variability of the sources investigated in that paper show many different patterns, like saw tooth, sine-function and intermittent. While the order of magnitude of the main period is about 200 days (Olech et al. 2019 found a range from 24 to 668 days), other longer periods are not excluded; one can see a slow decrease in amplitude in the upper panel, and an increase in the lower one. The masers are associated with HMYSOs (high masss young stellar objects). Due to these characteristics of the variability, it will take several years to accumulate sufficient data to look for meaningful correlations of the variability parameters with parameters of the HYMSOs hosts.

Furthermore, being massive stars, these objects are tracers of spiral arms and can play a major role in establishing the spiral structure of the Galaxy, by providing precise position and velocities of the arms. Such a work was carried by the Japanese-Korean program VERA (Oh et al. 2010). VERA is a VLBI network which has some similarities with IVIA in number and sizes of the antennas. The map of the structure of the Galaxy was partialy complemented by Reid et al. (2019). However since the VERA antennas are in the Northern hemisphere, they could not observe an important part of the Galactic disk (Figure 4). 


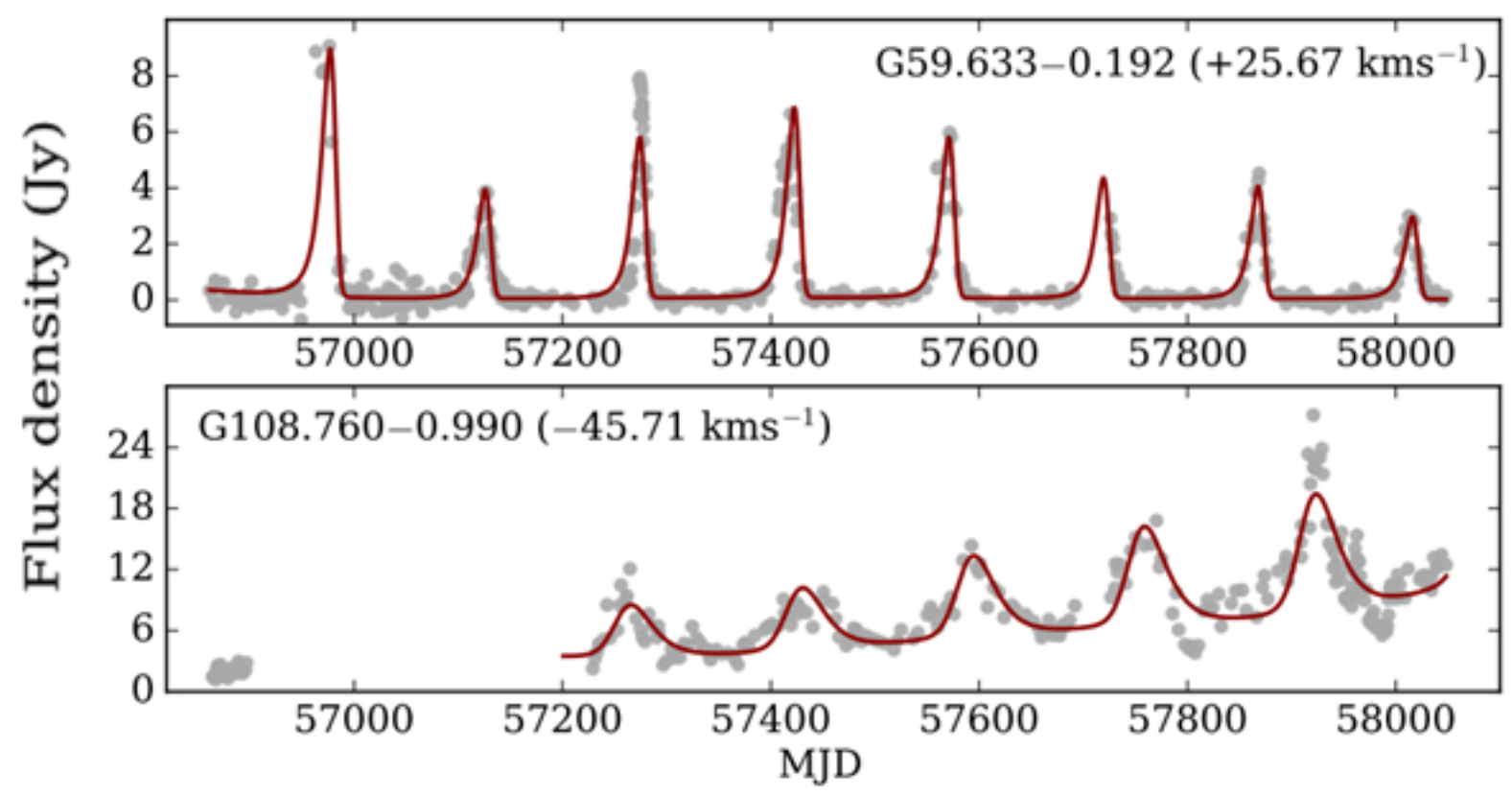

Figure 3. Time variability of two $6.668 \mathrm{GHz}$ methanol masers observed with the $32 \mathrm{~m}$ antenna of Torún (Poland) by Olech et al. (2019). The time is indicated in Julian days, the Galactic coordinates of the sources, as well as their velocities with respect to the Local Standard of Rest are indicated inside the figures.

An important program for the second phase of the IVIA project could be to obtain the distance of the methanol masers by means of parallax measurements using $\mathrm{VLBI}$, to determine the position of the spiral arms that can only be oberved from the South. Since the parallax is the oscillation in the position of the source due to the Earth orbit around the Sun, it requires several measurements along a period of one year to be measured. This may be difficult to achieve if the variability of the source looks like the upper panel of Figure 3. A preliminary observation program with single dish could be done to establish the variability properties of the masers, in preparation for the VLBI schedule of observations.

\section{Variability of Active Nuclei}

Other types of variability studies will also be performed in the first phase. One class of objetcs includes quasars, blazars and nuclei of Seyfert galaxies. An example of radio variability study being made by a Brazilian group is described by Beaklini et al. (2019).

These objects are Active Galactic Nuclei; some of them show variability at all the wavelengths observed (radio, infrared, optical, UV, $X$-ray and $\gamma$-ray). In radio, it would be important to monitor the active objects at well separated frequencies, in order to be able to measure the phase delays between the peaks of emission. This will be possible in near future using the IVIA single dish in Brazil, around 6 $\mathrm{GHz}$, the Pierre Kaufman observatory (former Itapetinga) at 22 and $43 \mathrm{GHz}$, and the LLAMA Argentinian-Brazilian telescope in construction in the Andes, at 200 and $600 \mathrm{GHz}$ (for instance). This type of monitoring provides important information on the physical processes occurring in these sources, which are still not fully understood (Antonucci 2013). 


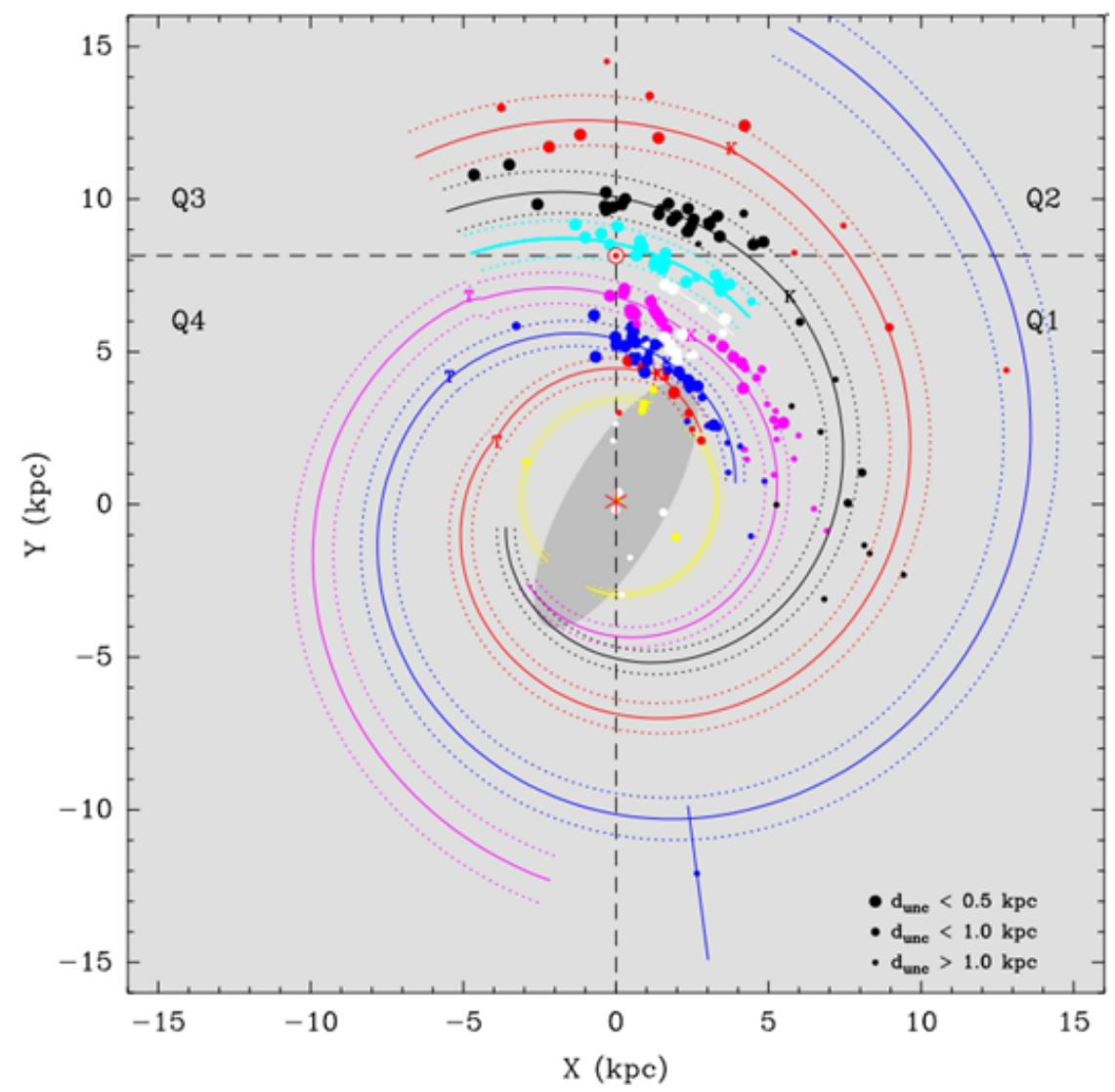

Figure 4. The spiral arms in the Galactic plane, and the maser sources which had their distances measured by VLBI parallax. The Sun is indicated by a red dot. The quadrants of the Galaxy are indicated by Q1, Q2, etc. (taken from Reid et al. 2019). One can see that there are much less VLBI measurements in Quadrant 3, which would require radiotelescopes in the Southern Sky.

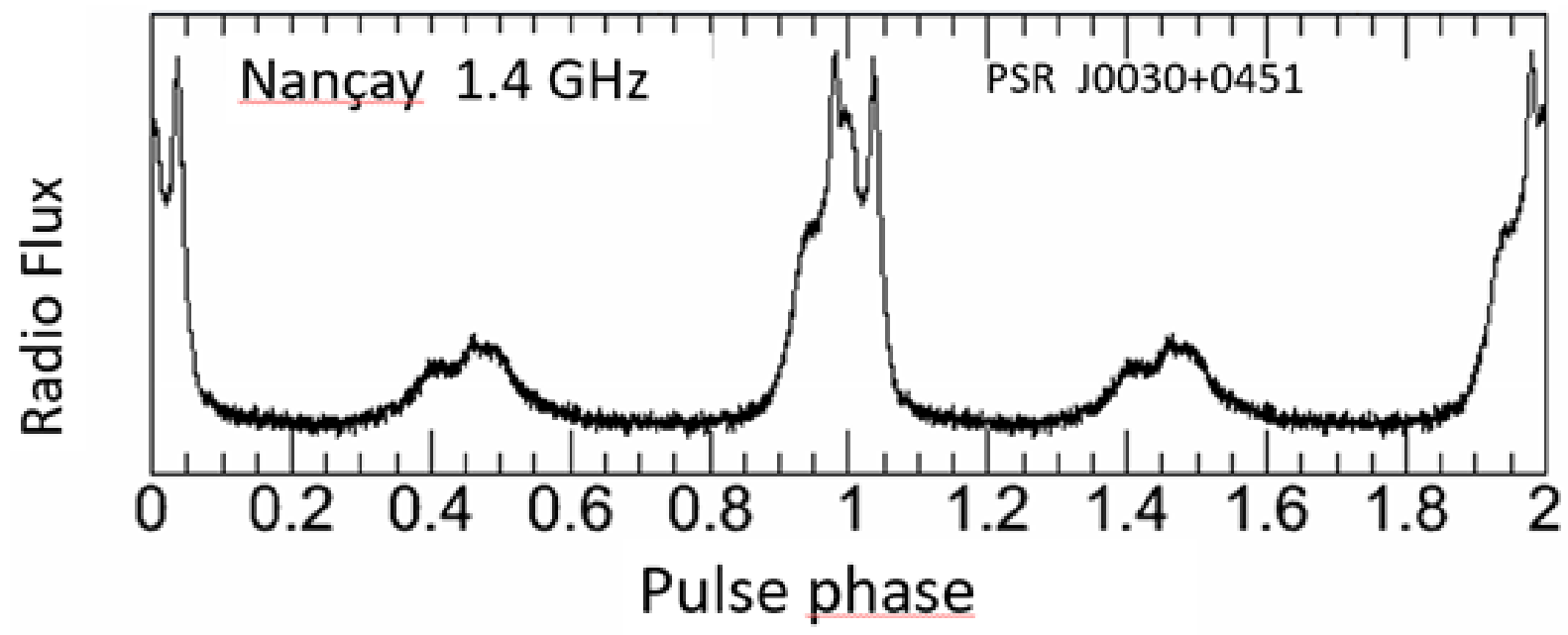

Figure 5. Light curve of the pulsar PSRJ0030+0451, a very fast pulsar (period $4.8 \mathrm{~ms}$ ) discovered in 2000 with the Arecibo radio telescope. The main pulse is in the center; two periods are shown along the time axis. 
Some of these objects will naturally be targets for VLBI observations in the second phase of IVIA activities. A few quasar jets, appear to expel like bubbles of plasma out of their nuclei at extremely high velocities, like ten times the speed of light, which seems to violate a fundamental statement of relativity. These are called "superluminal" motions. Abraham \& Romero (1999), working at IAG-USP, were pioneers in attributing these apparent motions to the precession of the jet, in the case of 3C273. What moves is not bubble of gas, but the position where the jet hits a distant cloud. They pointed out, in that paper, the importance of variability studies.

\section{Pulsars}

There is presently a renewed interest in pulsars. The investigation of pulsars, during the single dish phase, could focus the shape of the pulses. Since the exact periods of the pulsars are known, it is possible to add up the records of individual pulses, and to obtain their "shapes" or light curves with a good signal to noise ratio. An example of the shape of a pulse is shown in Figure 5, a result from the Nançay Observatory, France.

Usually there is a main pulse and a sub-pulse midway between the main pulses. Basically the pulses are due to the very fast rotation of neutron star, which presents a magnetic axis inclined with respect to the rotation axis. Typical inclinations are of the order of $20-30^{\circ}$. The rotation axis, on its turn, is usually not perpendicular to the line-of- sight to the Sun. Most of the radiation is emitted in the direction of the magnetic axis, which comes close to the line of sight once per turn. The secondary peak is supposed to originate from the other extremity of the magnetic axis, which also passes close to the line of sight once per turn. It is as if a lantern were attached at each magnetic pole, pointing outwards, and iluminating us once per turn. However, this simple classical model is not sufficient to explain many of the observed features (substructures, asymmetry of the poles, intensity variations, etc) and very little is known about the neutron stars. Quite complex models are being proposed, eg. Riley et al. (2019), who makes use of combinations of hot spots on the surface of the neutron star. This evolution of the pulsar field of research tells us there will be a need for many cases of well studied pulse shapes, observed in radio and also at other wavelengths, including $\gamma$-rays, to make progress.

In a way totally independent from the nature of the pulses, the pulsars are very interesting objects for studies of the structure of the Galaxy. They are remnants of supernova explosions, which means that their parent stars were quite massive. Massive stars are the best tracers of spiral arms. Deller et al. (2019) observed pulsars with VLBI, using the VLBA system in USA, and determined precise distances by parallax measurements for 57 of them. They present a map of the galactic plane where these objects are situated on spiral arms, and clearly delineate for instance, the Perseus arm. This survey suggests that in future IVIA could do similar VLBI observations, with the aim to improve the knowledge on the spiral structure of our Galaxy, in a region that cannot be observed by the VLBA (Galactic longitudes larger than $180^{\circ}$ ). This effort would be complementary with the one proposed above based on methanol maser VLBI observations. A preparation during the single dish phase could be done, to constitute a list of candidate pulsars with sufficient flux for the future VLBI observations.

Still concerning pulsars, there is even a proposal to attempt to detect gravitational waves in space, using them (Lommen 2015). The idea is simple. Consider that the pulsars are perfect clocks emitting pulses always with the same 
stable period. If a gravitational wave crosses the path of the pulse towards the Earth, it expands or contracts the space, and this will cause a delay or advance on the time of arrival of the pulses. What has to be done is to compare the arrival times with an atomic clok, in the hope to detect such variations. The result is not at all garanteed, as many years of monitoring have already passed without clear results.

\section{Fast Radio Bursts}

The Fast Radio Burst constitute a new and fascinating subject of research that we can include here, in this general topic of variability. At the beginning, such radio burst were so fast and desappeared totally, so that it was impossible to investigate them. Some researchers were wondering if they existed at all or were problems of the equipment. However, we have now at least one object that presented repeated fast bursts (Scholz et al. 2016). These bursts where observed by several radio telescopes, at different frequencies. This permitted to measure the difference of arrival times of a same burst at different frequencies, which is attributed to differences of propagation velocity of radio waves as a function of frequency, in the ionized interstellar medium. The path crossed by the signal inside our Galaxy would not be long enough to produce the observed differences in arrival times, so that it was concluded that the source is extra-galactic. This type of research requires monitoring regions of the sky where repeated burst could happen, and organize systems of alerts and observations at different frequencies.

\section{VLBI observation of asteroids}

We now report on the experiment of radar VLBI observations of the asteroid 2012 DA14 (Nechaeva et al. 2015). There have been many experiments of radar observations of Near-Earth asteroids, which are potentially dangerous, in the last decade. We select this one as an example because it involved several antennas similar to IVIA ones, situated at large distances one from the others. This method allows the simultaneous determination of the radial velocity, the distance to the object, its angular position (like $\alpha, \delta$ ), and proper motion, and can successfully be used to determine the orbits of near-Earth space objects. The experiment with asteroid 2012 DA14 consisted in sending a powerful $5 \mathrm{GHz}$ wave to irradiate the asteroid, using the RT-7O planetary radar antenna situated in Levpatoria, Crimea. The $5 \mathrm{Ghz}$ radiation reflected by the asteroid allowed to perform VLBI observations with a normal network of receiving antennas, with one in Latvia and two in Italy. The experiment was successful in determining several parameters of the asteroid. This may look like a too ambitious experiment, but it may happen that some of the IVIA antennas will be invited to participate to experiments leaded by countries which possess a radar antenna. There is interest in asteroid research in Brazil, remembering that Observatório Nacional/MCTI (Rio de Janeiro) maintains a $1 \mathrm{~m}$ robotic telescope in the North-East of Brasil, OASI, to observe small bodies of the solar system, and in particular the Near-Earth objects.

\section{Geodesy and Meteorology}

The VLBI technique is not only useful for astronomy. It allows to quantify the continental drifts, measuring distances between radiotelescopes with millimeter accuracy. VLBI contributes to determining the global terrestrial reference frame (ITRF). Being referenced to a kinematically non-rotating frame, it is the only technique to provides Earth Orientation Parameters, like polar motion, universal time, and celestial pole offsets. The rotational 
fluctuations of the Earth, responsible for slight variations in the length of a day, must be monitored and understood.

VLBI also allows to determine meteorological parameters from its observations. The tropospheric information obtained by VLBI over long periods provides important insight about the Earth's climate (Balidakis et al. 2018). Long-term climate and environmental evolutions are also observed due to the high accuracy of the VLBI results. For instance, variations in continental water storage produces loading deformations of the crust, with typical peak-to-peak variations at very long baseline interferometry (VLBI) sites of 3-15 $\mathrm{mm}$ in the vertical component and $1-2 \mathrm{~mm}$ in the horizontal component (Eriksson \& MacMillan 2014).

\section{CONCLUSIONS}

We have listed many attractive scientific possibilities for the use of the use of the IVIA antennas. There are already, in Brazil, researchers with interest and background in programs like methanol masers or quasar observations. These are quite easy to implement for the first phase with single dish.

The possibility to work in collaboration with the other IVIA countries, and also with African countries, exchanging experiences with them, is also very appealing. Scientific and technical coordination between the institutions involved, exchange of information and experiences, organization of regional workshops and schools, are among the expected benefits listed in the document of the IVIA foundation.

Thanks to the good will of Star One Company, the operation of one of the antenas, possibly the Tanguá antenna, will not be expensive. We need initial funds to refurbish the antena and construct a good receiver, but this will amount less than any other project of even a modest scientific telescope. There is no reason to refrain, thinking that Brasil is already involved in other important radio astronomical projects, like LLAMA and Bingo. There are synergies among the radio-astronomical projects, and most of the people involved are not the same. An idea previously proposed by Star One's director (A.F.G. Castro) to deploy remote control of the Brazilian IVIA antenna(s), will facilitate the use by different groups in Brazil, and will increase the interest in radio astronomy in general. VLBI is an unavoidable technique, which represents the top of the technological development in radio-astronomy, with new applications outside astronomy appearing every year, as we discussed in the Science Cases section. Big efforts are being made around the world. At the moment, Russia is developing a the space project Millimetron, aiming to put a $10 \mathrm{~m}$ radio telescope in space, to perform single-dish observations, but also VLBI with ground-based antennas (Kardashev et al. 2014). The Earth is not big enough, so that we have to put antennas outside the Earth to achieve long enough baselines. Large interferometers are being constructed in Australia (SKA) and in South Africa (Meerkat).

How can we understand that Brazil does not develop any strong activity in VLBI? And how can we understand that having the Star One antennas available, we do not use them, even for single dish astronomy?

The first phase of the IVIA project, is not an expensive one, and the Brazilian team is convinced that it will be possible to go on with it, in spite of the economical difficulties that are affecting mainly the federal institutes and universities, at the moment. The first official visit of the IVIA team to the Tanguá antenna is described in Apendix B. The technical conclusion is that the plan to rehabilitate the Tanguá antenna for radio astronomy is perfectly feasible. 


\section{REFERENCES}

ABRAHAM Z \& ROMERO GE. 1999. Beaming and precession in the inner jet of 3C 273. A\&A 344: 61-67.

ANTONUCCI R. 2013. Astrophysics: Quasars still defy explanation. Nature 495(7440): 165-167. doi:10.1038/495165a.

BALIDAKIS K, NILSSON T, ZUS F, GLASER S, HEINKELMANN R, DENG Z \& SCHUH H. 2018. Estimating Integrated Water Vapor Trends From VLBI, GPS, and Numerical Weather Models: Sensitivity to Tropospheric Parameterization. Journal of Geophysical Research (Atmospheres) 123(12): 6356-6372. doi:10.1029/2017JD028049.

BEAKLINI PPB, DOMINICI TP, ABRAHAM Z \& MOTTER JC. 2019. Multiwavelength analysis of brightness variations of $3 C$ 279: probing the relativistic jet structure and its evolution. A\&A 626: A78. doi:10.1051/0004-6361/201935170.

CLIVATI C ET AL. 2020. Common-clock very long baseline interferometry using a coherent optical fiber link. Optica 7(8): 1031. doi:10.1364/OPTICA.393356.

DELLER AT ET AL. 2019. Microarcsecond VLBI Pulsar Astrometry with PSR $\pi$ II. Parallax Distances for 57 Pulsars. ApJ 875(2): 100. doi:10.3847/1538-4357/ab11c7.

ERIKSSON D \& MACMILLAN D. 2012. Continental Hydrology Loading Observed by VLBI Measurements. In: Seventh General Meeting (GM2012) of the international VLBI Service for Geodesy and Astrometry (IVS), p. 415-419.

GAYLARD MJ ET AL. 2011. An African VLBI Network of radio telescopes. In: Proceedings of SAIP2011. p. 473-478.

KARDASHEV NS ET AL. 2014. Review of scientific topics for the Millimetron space observatory. Physics Uspekhi 57(12): 1199-1228. doi:10.3367/UFNe.0184.201412c.1319.

LOMMEN AN. 2015. Pulsar timing arrays: the promise of gravitational wave detection. Reports on Progress in Physics 78(12): 124901. doi:10.1088/00344885/78/12/124901.

NECHAEVA M ET AL. 2013. First Results of the VLBI Experiment on Radar Location of the Asteroid 2012 DA14. Baltic Astronomy 22: 341-346. doi:10.1515/astro-2017-0164.

OH CS, KOBAYASHI H, HONMA M, HIROTA T, SATO K \& UENO Y. 2010. VERA Observations of $\mathrm{H}_{2} \mathrm{O}$ Maser Sources in Three Massive Star-Forming Regions and Galactic Rotation Measurements. PASJ 62: 101. doi:10.1093/pasj/62.1.101.

OLECH $M$, SZYMCZAK $M$, WOLAK P, SARNIAK $R$ \& BARTKIEWICZ A. 2019. 6.7 GHz variability characteristics of new periodic methanol maser sources. MNRAS 486(1): 1236-1254. doi:10.1093/mnras/stz926.

REID MJ ET AL. 2019. Trigonometric Parallaxes of High-mass Star-forming Regions: Our View of the Milky Way. ApJ 885(2): 131. doi:10.3847/1538-4357/ab4a11.

RILEY TE ET AL. 2019. A NICER View of PSR J0030+0451: Millisecond Pulsar Parameter Estimation. ApJ 887(1): L21. doi:10.3847/2041-8213/ab481c.

SCHOLZ P ET AL. 2016. The Repeating Fast Radio Burst FRB 121102: Multi-wavelength Observations and Additional Bursts. ApJ 833(2): 177. doi:10.3847/1538-4357/833/2/177.

\section{How to cite}

LEPINE JRD ET AL. 2021. IVIA - Ibero-American VLBI Initiative -Progress on the Brazilian side. An Acad Bras Cienc 93: e20201697. DOI 10.1590/0001-3765202120201697.

Manuscript received on October 25, 2020;

accepted for publication on January 11, 2021

\section{JACQUES R.D. LEPINE ${ }^{1}$}

https://orcid.org/0000-0002-8969-0313

JEAN PIERRE RAULIN ${ }^{2}$

https://orcid.org/0000-0002-7501-3231

TANIA DOMINICl ${ }^{3}$

https://orcid.org/0000-0002-1522-0925

CARLOS GUILLERMO G. DE CASTRO ${ }^{2}$

https://orcid.org/0000-0002-8979-3582

FERNANDO VIRGILIO ROIG ${ }^{4}$

https://orcid.org/0000-0001-7059-5116

ANDRÉ WIERMANN ${ }^{4}$

https://orcid.org/0000-0001-5153-0886

MARCELO BORGES FERNANDES 4

https://orcid.org/0000-0001-5740-2914

YASUCHI HADANO ${ }^{2}$

https://orcid.org/0000-0001-9480-6600

FATIMA SALETE CORRERA 5

https://orcid.org/0000-0001-7826-088X

WESLEY BECCARO 5

https://orcid.org/0000-0001-6599-2344

MARCOS AURELIO LUQUEZE 5

https://orcid.org/0000-0002-5835-4069

ANTONIO SANDRO VERRI ${ }^{5}$

https://orcid.org/0000-0003-2956-3408

DANILO CESAR ZANELLA ${ }^{1}$

https://orcid.org/0000-0003-0343-9062

KARIN MENÉNDEZ-DELMESTRE 6

https://orcid.org/0000-0003-3153-5123 
THIAGO SIGNORINI GONÇALVES 6

https:// orcid.org/0000-0003-2374-366X

ULISSES BARRES DE ALMEIDA ${ }^{7}$

https://orcid.org/0000-0001-7909-588X

\section{MILITÃO VIEIRA FIGUEREDO ${ }^{8}$}

https://orcid.org/0000-0003-2784-0678

${ }^{1}$ Universidade de São Paulo, Instituto de Astronomia, Geofísica e Ciências Atmosféricas, Rua do Matão 1226, Cidade Universitária, 05508-090 São Paulo, SP, Brazil

${ }^{2}$ Universidade Presbiteriana Mackenzie, Rua da Consolação, 930, Consolação, 01302-907 São Paulo, SP, Brazil

${ }^{3}$ Museu de Astronomia e Ciências Afins, Ministério da Ciência, Tecnologia e Inovações (MAST/MCTI), Rua General Bruce 586, Bairro Imperial de São Cristóvão, 20921-030 Rio de Janeiro, RJ, Brazil

4Observatório Nacional (OB/MCTI), Rua General José Cristino, 77, São Cristóvão, 20921-400 Rio de Janeiro, RJ, Brazil

${ }^{5}$ Escola Politécnica, Universidade de São Paulo, Av. Prof. Luciano Gualberto, trv. 3, no 158, 05508-010 São Paulo, SP, Brazil

${ }^{6}$ Universidade Federal do Rio de Janeiro (UFRJ), Observatório do Valongo, Ladeira Pedro Antônio, 43, 20080-09o Rio de Janeiro, RJ, Brazil

${ }^{7}$ Centro Brasileiro de Pesquisas Físicas (CBPF/MCTI), Rua Dr. Xavier Sigaud, 150, Urca, 22290-180 Rio de Janeiro, RJ, Brazil

${ }^{8}$ Universidade Federal do Vale do São Francisco (UNIVASF), Av. José de Sá Maniçoba, s/n, Centro, 56304-917 Petrolina, PE, Brazil

Correspondence to: Jacques R.D. Lepine

E-mail: jacques.lepine@iag.usp.br

\section{(cc) BY}




\section{APPENDIX A - INSTITUTIONS AND PEOPLE INVOLVED IN IVIA}

- Instituto de Radioastronomía y Astrofísica, Universidad Nacional Autónoma de México (Laurent Loinard, Stan Kurtz, Gisela Ortiz León);

- Centro de Investigaciones Espaciales, Universidad de Costa Rica (Carolina Salas Matamoros);

- Observatorio Astronómico de Córdoba, Argentina (Carlos Valotto);

- Facultad de Ingenieria Electrica y Electronica, Universidad Nacional del Centro del Perú (José Kaname Ishitsuka Iba). It is with great regret that we communicate that our dear colleague José Ishitsuka passed on 16 December 2020;

- Universidad ECCl, Bogotá, Colombia (Germán Chaparro);

- Oficina Regional Andina de Astronomía para el Desarrollo, Bogotá, Colombia;

- Universidade de São Paulo, Brazil (Jacques Lepine);

- Ministerio de Industria, Energía y Minería, Uruguay (Manuel Caldas);

- Observatorio Astronómico de Quito de la Escuela Politécnica Nacional, Ecuador (Ericson López);

- Instituto Geográfico Nacional, Ministerio de Fomento, España (Jose Antonio Lopez Fernandez);

- Instituto de Telecomunicações, Aveiro, Portugal: (Domingos Barbosa, Sonia Anton, Dalmiro Maia);

- Joint Institute for VLBI ERIC (Headquarters in Netherlands, Francisco Colomer)
- Estación Terrena de Areguá (Paraguay, Guillermo Giménez de Castro, Diego Stalder). This institution joined in early 2020.

\section{APPENDIX B - TECHNICAL ASPECTS}

\section{I- Visit to the Tanguá antenna}

In the first visit to the Tanguá antenna in September 2019, the IVIA team was composed of: Fernando Roig, Jacques Lepine, Jean Pierre Raulin, Tania Dominici, Yasuchi (Rubens) Hadano, André Wiermann, Carlos Fermino (mechanical engineer consultant) The team was welcomed by Star One company engineers: Severino Lucena and Marcos Garcia.

The place has a large well-kept green area and many antennas (we counted 20), some still being used to receive satellite data. Tanguá Station serves as a back-up to Guaratiba main station, Rio de Janeiro, where the submarine cables also arrive. Only one of the two large antennas, Tanguá 2, is usable; the other one, older and a little smaller, is deteriorated.

According to Eng. Severino Lucena, who guided the visit, there is no problem of radio interference, because the the site is protected by mountains and protective laws.

Tanguá 2, manufactured by the US company ITT, was inaugurated in 1972. It has a dish reflector made of aluminum panels (Figure 1), and alt-azimuthal mounting. The azimuth movement is ensured by sliding the antenna, which rolls over a large ring of steel.

The panels of the antena are in good condition but dirty. The problem is similar to that reported by Mexican colleagues for the Tulancingo antenna; it is easy to wash them.

The light metal frame of the reflector plate features rusty beams, including a beam already detached on one side. This affects practically only the outer edge of the dish. The outer beams 


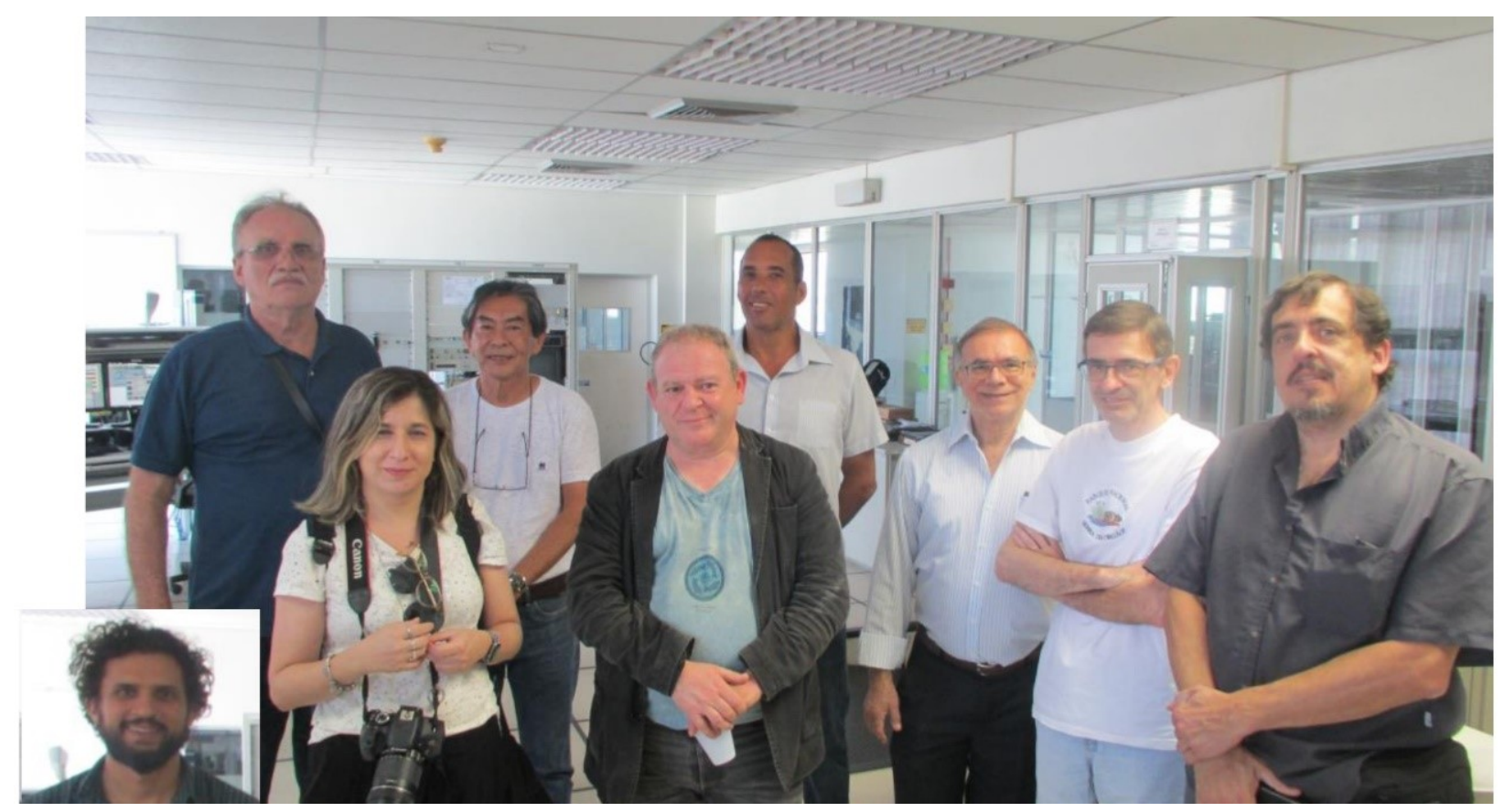

Figure 6. Technical visit to the Tanguá antenna in "Estacao Terrena da Embratel" on September 19, 2019.

would have to be replaced to avoid further damage.

The heavier steel structure of the antenna has numerous points of rust. However, this rust does not endanger the structure. It is a matter of cleaning and painting to prevent rust from spreading.

It was reported by Star One that the steel ring on which the antenna rolls for azimuthal motion has a depression at one point, because the antenna has been pointing to the same sky position for years. This defect does not appear at a simple glance. It would be important to better characterize the depression in length and depth. There are solutions.

Azimuth movement is performed by two sets of motors, that act on the wheels that rolls on a ring-shaped rail which is fixed on the concrete base. Each set has two motors, one for higher speeds and one smaller for slower speeds. It would be necessary to do maintenance of the motors, replace bearings and brushes. A possibility is to replace the engines by more modern ones. Elevation handling is ensured by a gear system which does not present serious damage, only surface oxidation. Only engine maintenance is required.

There is an encoder on each axis. We didn't see the azimuth encoder. The elevation encoder was easily accessible along the elevation axis. Carlos Fermino from eFe startup company recommends to replace them. The encoder resolution is 1/1000 degrees, or 3.6 arc seconds, which is quite sufficient. A $32 \mathrm{~m}$ antenna operating at $6 \mathrm{GHz}(\lambda=5 \mathrm{~cm})$ has a 5.3 minute arc beam, similar to the Itapetinga antenna beam at $22 \mathrm{GHz}$, which is nice to compare the same sources at different frequencies.

The radio wave is carried from top to bottom with 4 reflections. It is necessary to evaluate the optics/mirrors. The 2 circular polarizations arrive at the bottom, which would allow to measure the polarization of the sources.

Under the antenna, the place where receivers are installed is very comfortable to work. Two cylindrical waveguides arrive, one for 4 
$\mathrm{GHz}$ (downlink) and one for $6 \mathrm{Ghz}$ (uplink), which correspond to the reception and transmission of satellites.

The antenna position control system is located in a large room in the main building. Real "tracking" of a radio source is not available; an order can be sent to position at a particular position, but not to follow a source. One could simulate a tracking motion by sending many successive positions. Such solution is not satisfactory to us; we have to implement a true tracking program. Since there are already several converted antennas in the IVIA collaboration, we could possibly obtain a program from a partner and adapt it to our case. We should perhaps have our own tracking computer, instead of using the one which is available.

\section{II- Receivers and time reference}

Our plan is to start using a simple room temperature amplifier with 4 to $8 \mathrm{GHz}$ bandwith (as agreed with the other IVIA partners) limited by filters, directly coupled to the output at the bottom of the existing horn system. The output of the amplifier goes to a total power detector, then to an analog to digital converter and to the data acquisition computer. We already tested such a simple receiver system and it worked; we should improve it and exchange ideas with the partners. For line observations we think of a Fast Fourier Transform system, that could be acquired or "home" made.

For the later VLBI phase, a different amplification system will have to be developped and a data storage system acquired. The most expensive part is the atomic clock. A hydrogen maser clock costs of the order of $300 \mathrm{~K} \mathrm{USD}$. However, there are alternative solutions. In an experiment made in Italy (Clivatti et al. 2020), the same hydrogen maser clock signal was delivered to two distant radio telescope sites using optical fibers, and successful VLBI observations were made. The total length of fibers used was 1739 $\mathrm{km}$. This suggests that we could deliver the maser clock signal available at observatório Nacional in Rio de Janeiro to Tanguá and even to Morungaba (São Paulo State), possibly using existing fiber links.

\section{III- Cooperation intention}

It has been reiterated by Star One that there is great interest in the reuse of antennas for science. They would bear local expenses (security, electricity, etc.), but we would be responsible for antenna refurbishing expenses. A very preliminary estimate discussed with Eng. Severino Lucena is that the total amount of repairs needed would be in the order of 500000 reais (about 100 k USD) without receivers. The cost of the antenna is estimated at 15 millions USD.

Eng. Lucena insisted that we should not take any decision without first seeing the Morungaba antennas made by NEC (Japanese company) that he thinks are in better condition. He recalled the idea previously suggested by Star One's director (A.F.G. Castro) to deploy remote control. It would be a breakthrough for youth participation across the country, and it is not too difficult to implement. 